OPEN ACCESS

Edited by:

Dongsheng Zhou,

Beijing Institute of Microbiology and Epidemiology, China

Reviewed by:

Qin Ma,

South Dakota State University,

United States

Feng Gao,

Tianjin University, China

Lei Zhou,

University of Florida, United States

${ }^{*}$ Correspondence:

Zongde Zhang

zzd417@163.com

Fei Chen

chenfei@big.ac.cn

${ }^{\dagger}$ These authors have contributed equally to this work.

Specialty section:

This article was submitted to Infectious Diseases,

a section of the journal

Frontiers in Microbiology

Received: 17 February 2017

Accepted: 26 May 2017

Published: 12 June 2017

Citation:

Lv L, Li C, Zhang X, Ding N, Cao T,

Jia $X$, Wang J, Pan L, Jia H, Li Z, Zhang J, Chen F and Zhang $Z$

(2017) RNA Profiling Analysis of the

Serum Exosomes Derived from

Patients with Active and Latent

Mycobacterium tuberculosis Infection.

Front. Microbiol. 8:1051.

doi: 10.3389/fmicb.2017.01051

\section{RNA Profiling Analysis of the Serum Exosomes Derived from Patients with Active and Latent Mycobacterium tuberculosis Infection}

\author{
Lingna Lv't, Cuidan $\mathrm{Li}^{2,3 t}$, Xiuli Zhang ${ }^{2,3 t}$, Nan Ding ${ }^{2 t}$, Tianshu Cao ${ }^{2 \dagger}$, Xinmiao Jia ${ }^{2,3}$, \\ Jinghui Wang' ${ }^{1}$ Liping Pan', Hongyan Jia', Zihui Li', Ju Zhang ${ }^{2}$, Fei Chen ${ }^{2,3,4,5 *}$ and \\ Zongde Zhang ${ }^{1 *}$
}

\begin{abstract}
' Beijing Key Laboratory for Drug Resistant Tuberculosis Research, Beijing Tuberculosis and Thoracic Tumor Research Institute, Beijing Chest Hospital, Capital Medical University, Beijing, China, ${ }^{2}$ CAS Key Laboratory of Genome Science and Information, Beijing Institute of Genomics, Chinese Academy of Sciences, Beijing, China, ${ }^{3}$ University of Chinese Academy of Sciences, Beijing, China, ${ }^{4}$ Collaborative Innovation Center for Genetics and Development, Shanghai, China, ${ }^{5}$ Sino-Danish College, University of Chinese Academy of Sciences, Beijing, China
\end{abstract}

Tuberculosis (TB) has exceeded HIV as the most lethal infectious disease globally for two consecutive years. Moreover, one third of the world's population is estimated to have latent tuberculosis infection (LTBI). This is mainly because of difficulties associated with diagnosis and treatment for both TB and LTBI patients. Exosomes provide a promising research tool for TB diagnosis and treatment because they are released from various cells containing valuable biochemical information related to disease. In this study, we performed RNA-sequencing analysis on exosomes derived from clinical specimens of healthy controls $(\mathrm{HC})$, active tuberculosis (ATB), and LTBI patients. Our results revealed the distinct gene expression profiles of the exosomes from LTBI and ATB patients. (1) We identified many distinct up-regulated and down-regulated differentially expressed genes (DEGs) in LTBI and ATB samples, and further screened the top-20 DEGs which might provide a potential panel for differentiation of HC, LTBI, and ATB. (2) We classified all the DEGs into six expression patterns, screened the top-20 genes in each pattern, and mainly focused on those highly expressed in LTBI and ATB. (3) Some Mycobacterium tuberculosis (Mtb) RNAs were only enriched in the exosomes of LTBI samples. (4) Pathway and function analysis further indicated down-regulated signaling pathways/immune response and up-regulated apoptosis/necrosis. Our findings indicate the selective packaging of RNA cargoes into exosomes under different stages of Mtb infection, while facilitating the development of potential targets for the diagnosis, prevention and treatment of tuberculosis. Keywords: tuberculosis (TB), Mycobacterium tuberculosis (Mtb), exosome, RNA sequencing, latent tuberculosis
infection (LTBI), transcriptome, biomarker, IPA

Abbreviations: ATB patient, active tuberculosis patient; DEG, differentially expressed gene; IGRA, interferon-gamma release assay; LTBI patient, latent tuberculosis infection patient; NTM, non-tuberculous mycobacteria; TST, tuberculin skin test. 


\section{INTRODUCTION}

According to the 2015 and 2016 WHO reports, tuberculosis has exceeded HIV as the most lethal infectious disease globally for two consecutive years (2014 and 2015). This is mainly because of difficulties associated with diagnosis and treatment of TB patients. In addition, elimination of tuberculosis has been partly prevented by the ability of $M t b$ to remain dormant in the human body for years without causing disease, a state referred to as LTBI (Velayati et al., 2016). An estimated 2 billion people are latently infected with $M t b$. Of these, $10 \%$ of infected individuals will develop active TB during their lifetime (Tufarielloa et al., 2003). Although the majority of infected individuals display no symptoms of disease, they develop a strong acquired immune response to the pathogen (Gideon and Flynn, 2011). Thus, early diagnosis of LTBI patients plays a crucial and important role in reducing morbidity, while also preventing onward transmission to susceptible individuals.

Exosomes provide a promising research tool for TB diagnosis and treatment because they are released from various cells containing valuable biochemical information (proteins, lipids, and nucleic acids) relating to disease, while facilitating cellcell communication by shuttling various molecules from donor to recipient cells. These membrane-enclosed vesicles (range in size from 30 to $150 \mathrm{~nm}$ ) play important roles in signal transduction (e.g., immune regulation) (Alipoor et al., 2016), material transportation (nucleic acids, proteins, lipids, and other biochemicals) (Théry, 2011), and cellular "trash bags" for elimination of excess intracellular substances (Subramanian et al., 2016).

Proteomic analysis has revealed many host proteins in addition to $41 \mathrm{Mtb}$ proteins within exosomes secreted by macrophages infected with either live $M t b$ or $M t b$ culture filtrate (Giri et al., 2010). Subsequent studies identified $20 \mathrm{Mtb}$ proteins in exosomes isolated from the serum of TB patients, including the antigens $85 \mathrm{~b}$, BfrB, GlcB, and Mpt64 (KruhGarcia et al., 2014). In addition, exosomes derived from the macrophages infected with Mycobacterium avium contained bacterial pathogenic glycopeptidolipids (Bhatnagar and Schorey, 2007). The extracellular exosomes carrying mycobacterial constituents have been reported to affect recipient cells by either silencing or promoting the immune responses. For example, exosomes released from infected macrophages stimulated naïve macrophages to produce proinflammatory TNF- $\alpha$, RANTES, and inducible nitric oxide synthase (iNOS) (Bhatnagar and Schorey, 2007; Bhatnagar et al., 2007). Conversely, in some cases, exosomes released from $M t b$-infected cells exhibited an inhibitory effect on IFN- $\gamma$-mediated activation of naïve macrophages (Singh et al., 2011).

Exosomes have also been found to contain various RNAs, including mRNA, rRNA, microRNA, and long non-coding RNA (lncRNA) (Gusachenko et al., 2013). Previous studies have indicated that the exosomes could transfer mRNAs to exchange phenotypic features between cells (Valadi et al., 2007). Singh et al. (2015) discovered some potential biomarkers (human miRNAs and mRNAs as well as mycobacterial RNAs in exosomes) that could be used to detect TB in patients. However, systematic exosomal RNA sequencing analysis in human clinical specimens of ATB and LTBI patients is still lacking.

In this research, we performed systematic RNA profiling analysis of the exosomal RNAs derived from the serum samples of HC, LTBI, and ATB individuals. Our results revealed distinct gene expression panels and patterns of the exosomes, indicating the selective packaging of RNA cargoes into exosomes under different physiological status. Pathway and functional analysis further indicated a gradual increase in deteriorated healthy signals in LTBI and ATB samples, including down-regulated signaling pathways/immune response, and up-regulated apoptosis/necrosis. These results provide key information on exosomes during the $M t b$ infectious process, and provide insight into the development of potential biomarkers using exosomal RNAs in TB diagnosis.

\section{MATERIALS AND METHODS}

\section{Study Participants}

This study was carried out in accordance with the recommendations of the Helsinki Declaration and its later amendments or comparable ethical standards, the Ethics Committee of the Beijing Chest Hospital, Capital Medical University with written informed consent from all subjects. All subjects gave written informed consent in accordance with the Declaration of Helsinki. The protocol was approved by the Ethics Committee of the Beijing Chest Hospital, Capital Medical University. All participants were at least 18 years old, HIV-negative and written, informed consent was obtained from these patients. TB patients were classified by their clinical presentation being consistent with $\mathrm{TB}$ infection, a positive $M t b$ culture, and a positive smear. Patients were excluded if they had a previous TB history or had received anti-TB therapy before enrollment. Latently infected subjects were defined as having a positive TST and IGRA using T-SPOT.TB (Oxford Immunotec, Abingdon, United Kingdom), normal chest computed tomography (CT), absence of clinical symptoms or evidence of active TB and other non-tuberculosis respiratory infections. The TST/IGRA two-step strategy is used because confirmatory IGRA is able to highly reduce the false positivity rate due to BCG vaccination or NTM infection in the initial TST. Healthy uninfected controls were enrolled with negative TST and T-SPOT.TB tests, normal chest CT and no clinical evidence of any diseases. The participant demographic information is shown in Table 1.

\section{Sample Preparation}

A total of 90 serum samples were collected. The serum samples were grouped according to the clinical cohort as ATB, LTBI, and healthy control (HC). Serum was obtained from each participant and then pooled based on the group (pooled $n=15$ for HC, LTBI, and ATB, respectively; $1 \mathrm{~mL}$ of serum in each pooled sample). For each group, two biological replicate pools were prepared (i.e., $n=30$ patients in total for HC, LTBI, and ATB, respectively). 
TABLE 1 | Clinical data of the participants.

\begin{tabular}{|c|c|c|c|c|c|c|}
\hline & HC 1 & HC 2 & LTBI 1 & LTBI 2 & ATB 1 & ATB 2 \\
\hline$N$ & 15 & 15 & 15 & 15 & 15 & 15 \\
\hline Male/Female & $9 / 6$ & $7 / 8$ & $6 / 9$ & $7 / 8$ & $11 / 4$ & $12 / 3$ \\
\hline Mean age $\pm S D(y)$ & $41.1 \pm 8.2$ & $43.4 \pm 8.6$ & $42.2 \pm 8.2$ & $40.0 \pm 7.9$ & $41.5 \pm 8.9$ & $42.6 \pm 9.6$ \\
\hline Age range $(y)$ & $22-56$ & $24-59$ & $25-55$ & $25-58$ & $20-65$ & $19-65$ \\
\hline Smokers/Non-smokers & $1 / 14$ & $5 / 10$ & $3 / 12$ & $5 / 10$ & $5 / 10$ & $6 / 9$ \\
\hline
\end{tabular}

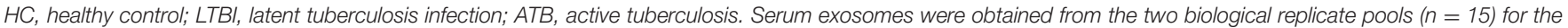
healthy control (HC), LTBI, and ATB samples.

\section{Exosome Isolation and RNA Extraction}

Exosome isolation from pooled serum samples was conducted as previously described. Briefly, the cell debris were removed by differential centrifugation at $1000 \times g$ for $10 \mathrm{~min}$ at $4^{\circ} \mathrm{C}$, and $16,500 \times g$ for $30 \mathrm{~min}$ at $4^{\circ} \mathrm{C}$, followed by ultrafiltration (through a $0.22 \mu \mathrm{m}$ filter; Millipore, Billerica, MA, United States). Then, the exosome pellet was obtained by ultracentrifugation at $120,000 \times g$ for $2 \mathrm{~h}$ and washed with PBS (Supplementary Figure 1). Isolated exosomes were immediately used for total RNA extraction using RNAiso-Plus (TaKaRa, Dalian, China) according to the manufacturer's instructions. RNA concentration was measured using Qubit ${ }^{\circledR}$ RNA Assay Kit in Qubit $^{\circledR} 2.0$ Fluorometer (Life Technologies, Camarillo, CA, United States). RNA integrity was assessed using the RNA Nano 6000 Assay Kit of the Agilent Bioanalyzer 2100 system (Agilent Technologies, Santa Clara, CA, United States).

\section{Sequencing and Data Processing}

Sequencing libraries were generated using NEBNext ${ }^{\circledR}$ Ultra $^{\mathrm{TM}}$ Directional RNA Library Prep Kit for Illumina ${ }^{\circledR}$ (NEB, Ipswich, MA, United States) following manufacturer's recommendations and index codes were added to attribute sequences to each sample. The library quality was assessed on the Agilent Bioanalyzer 2100 system (Agilent Technologies, Santa Clara, CA, United States). The RNA libraries were sequenced on the Illumina Hiseq 2500 Genome Analyzer platform in pair-end mode.

For each sample, the mRNA sequencing reads were aligned to the UCSC human reference genome (hg38) using Tophat (version 2.0.9) (Langmead et al., 2009; Trapnell et al., 2009) with default parameters. The aligned reads were further subjected to Cufflinks (version 2.2.1) (Trapnell et al., 2010) software for assembling transcripts with the parameter "-M" for filtering reads mapped on ribosomal RNAs. DEGs were generated using Cuffdiff (version 2.2.1) (Trapnell et al., 2010). The GTF annotation file was downloaded from the GENCODE (version 25) website for transcript assembling and gene annotation. Unsupervised hierarchical clustering of genome-wide expression profiles (read count $>1$ ) was performed using the pheatmap ( $\mathrm{R}$ software). Gene Ontology (GO) function enrichment was generated by both DAVID (version 6.8) (Huang et al., 2009a,b). We also performed core analysis and comparison analysis using the Ingenuity Pathway Analysis (IPA) software ${ }^{1}$ to obtain canonical

\footnotetext{
${ }^{1}$ https://www.qiagenbioinformatics.com
}

pathway and disease and bio functions, which were evaluated by $p$-value $(p$-value $<0.05)$ and $Z$-score $(Z$-score $\neq 0)$. Sequence data is now available on the Gene Expression Omnibus (GEO; accession number: GSE94907).

\section{RESULTS}

\section{Expression Profiles of Serum Exosomes in HC, LTBI, and ATB Individuals}

By mapping to the human reference genome (hg38), we obtained the gene expression profiles of serum exosomes. A total of 44187 (HC), 43428 (LTBI), and 44261 (ATB) expressed genes were identified, of which 18913,18882 , and 18926 coding genes were obtained from HC, LTBI, and ATB patients, respectively (Supplementary Table 1). As shown in Figure 1A, many types of RNAs, including mRNAs, pseudogenes, and lncRNAs, were secreted into the exosomes, which shared a similar proportion of genes in each sample (HC, LTBI, and ATB).

As for the expression level, the coding genes of LTBI had higher expression levels than those of HC and ATB (Figure 1B). On the contrary, pseudogenes had lower expression levels in serum exosomes of HC than in LTBI and ATB samples. Specifically, unprocessed pseudogenes had slightly higher expression levels in ATB patients than in LTBI patients. In addition, IncRNAs in serum exosomes of LTBI exhibited higher expression levels compared with HC and ATB samples. Although little is known of how lncRNAs are packaged into exosomes, previous studies have suggested that exosomal lncRNAs had phenotypic effects within the recipient cells (Kogure et al., 2013).

To obtain the differential expression profiles among the serum exosomes in the HC, LTBI, and ATB individuals, we selected the DEGs by pairwise comparison (fold change $\geq 2$ and $p$-value $<0.05$ ), and plotted a heatmap (Figure 2A). The results revealed the distinct gene expression profiles of the exosomes for the HC, LTBI, and ATB patients: 1188 genes were highly expressed in HC samples compared with LTBI and ATB samples (the left column of Figure 2A). In contrast to the HC and ATB samples, 1020 genes (the middle column) were highly expressed in LTBI samples, while 681 genes (the right column) were highly expressed in ATB samples compared with the other two samples.

Further GO functional analysis indicated different enrichments of GO categories in the three samples (Figure 2B). Among them, 12 and 14 genes in ATB samples displayed 


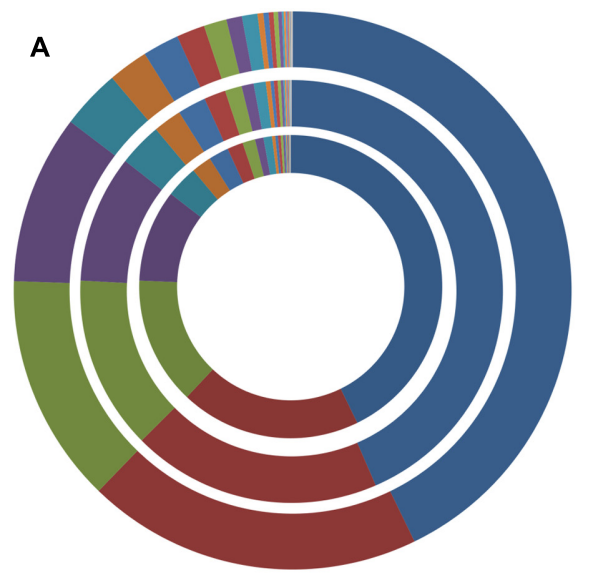

\begin{tabular}{|c|c|}
\hline $\begin{array}{l}\text { lincRNA } \\
\text { unprocessed pseudogene } \\
\text { TEC } \\
\text { transcribed unprocessed pseudoger } \\
\text { processed_transcript } \\
\text { IG_V_pseudogene } \\
\text { IG_V_gene } \\
\text { transcribed_unitary_pseudogene } \\
\text { snRNA } \\
\text { TR_V_pseudogene } \\
\text { 3prime_overlapping_ncRNA } \\
\text { IG_C_pseudogene } \\
\text { scaRNA } \\
\text { bidirectional_promoter_IncRNA } \\
\text { IG_J_gene }\end{array}$ & 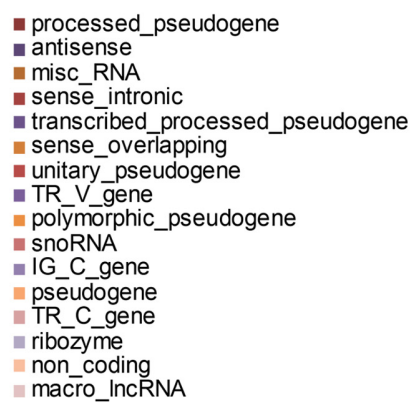 \\
\hline
\end{tabular}

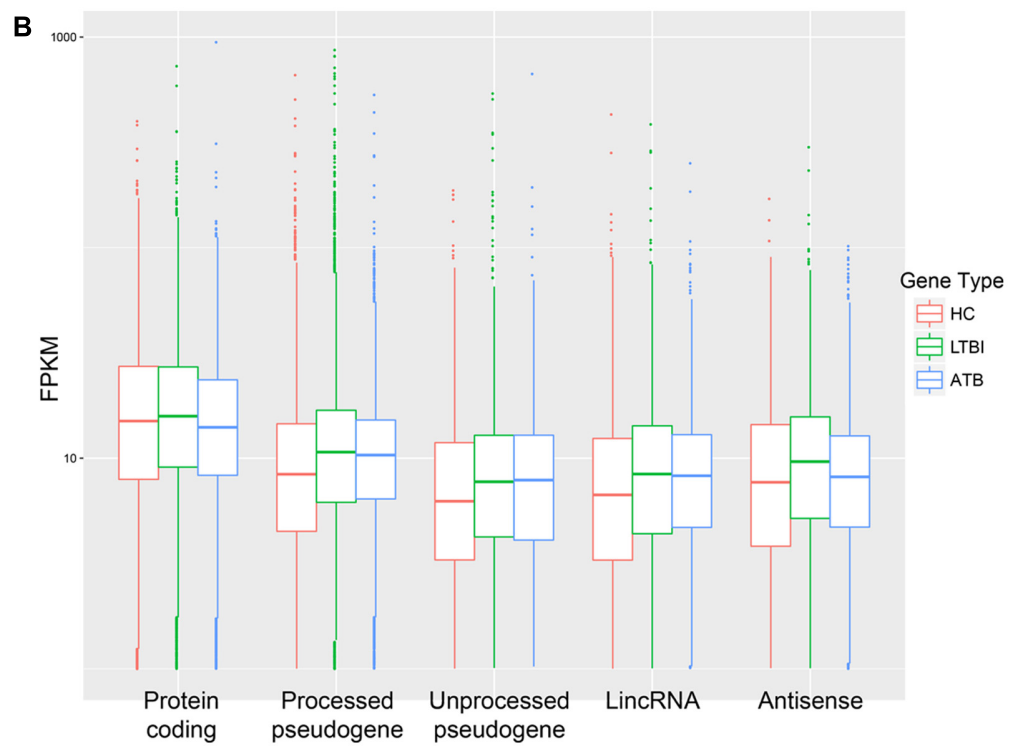

FIGURE 1 | General gene expression profiles among healthy control (HC), LTBI, and ATB samples. (A) Many types of RNAs were secreted into the exosomes (shown in different colors). They also shared a similar proportion of gene number in each sample (circles from outer to inner represent $\mathrm{HC}$, LTBI, and ATB). (B) The FPKM values of top-five types of RNAs in the three samples.

enrichments in "lipid metabolism" and "extracellular matrix organization" GO categories. Previous studies have indicated that $M t b$ can survive in macrophages using lipid from host cells (Ouellet et al., 2011). Therefore, the up-regulation of lipid metabolic genes in ATB exosomes might accelerate the transfer of signaling substances to facilitate the survival of $M t b$ in ATB patients. On the other hand, three of the 14 up-regulated extracellular matrix genes coded for three collagens $(19 \alpha 1,1 \alpha 2$, and $11 \alpha 1$ ), which have been reported to help form granuloma (Kaarteenaho-Wiik et al., 2007; Shammari et al., 2015).

\section{Expression Panels of Serum Exosomes in HC, LTBI, and ATB Individuals}

In comparison with the HC samples, we identified 769 up-regulated genes and 643 down-regulated genes in the LTBI individuals, while these numbers increased to 999 (up-regulated) and 1582 (down-regulated) in ATB individuals (Figure $\mathbf{3 A}$ and Supplementary Table 2). To further investigate the detailed differences in DEGs between LTBI and ATB samples, we overlapped these using a Venn diagram (Figure 3B). They exhibited relatively distinct expression panels: 598 and 833 genes were only up-regulated and expressed in LTBI and ATB, respectively; 338 and 1272 genes were uniquely downregulated and expressed in LTBI and ATB, respectively; while 5 genes were up-regulated and expressed in LTBI but down-regulated and expressed in ATB. In addition, there was a small portion of DEGs (fold change $>2$ and $p$-value $<0.05)$ in ATB and LTBI that shared similar expression panels (166: up-regulation; 305: down-regulation). 
A

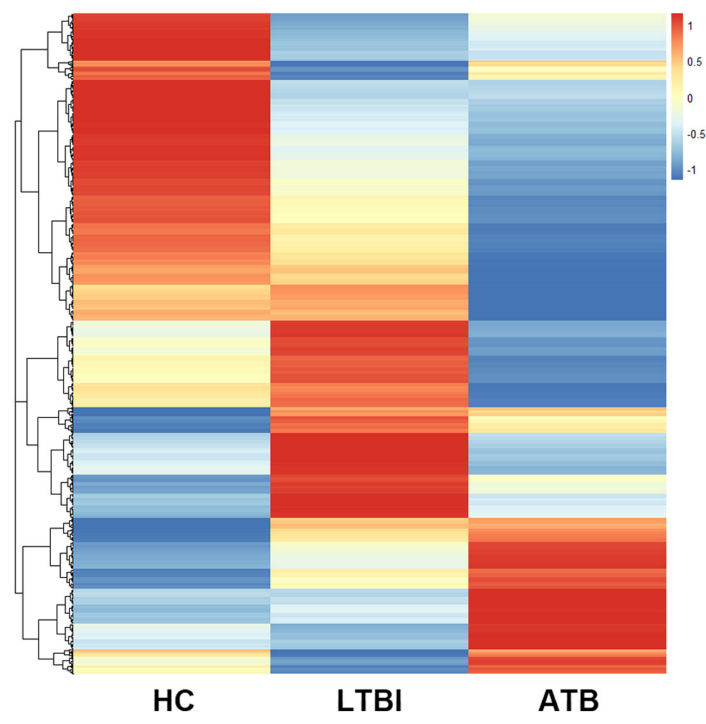

B negative regulation of ubiquitin-protein ligase activity involved in mitotic cell cyclechromosome segregationresponse to nutrienlipid metabolic process-

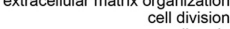
cell cycle-
otic processnscription from RNA polymerase II promoteative regulation of transcription, DNA-templatenegative regulation of transcription from RNA polymerase II promoter-
positive regulation of transcription from RNA polymerase II promoter-
transcription, DNA-templatedmovement of cell or subcellular componen osteoblast differentiationpositive regulation of defense response to virus by hoscellular protein metabolic proces negative regulation of gene expression SRP-dependent cotranslational protein targeting to membranepositive regulation of ERK1 and ERK2 cascade$\begin{array}{cc}\text { positive regulation of ERK1 and ERK2 cascade- } & \text { viral transcription- } \\ \text { positive regulation of cell migration- } & \text { LTBI } \\ \text { nuclear-transcribed mRNA catabolic process, nonsense-mediated decay- } & \text { rRNA processing- }\end{array}$

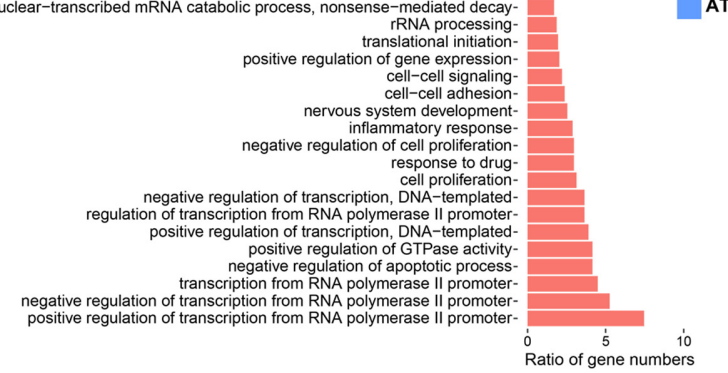

FIGURE 2 | Profiles of DEGs among HC, LTBI, and ATB samples. (A) Heatmap of DEGs among the three samples. (B) The Gene Ontology (GO) biological process categories of the top 30 percentage of the DEGs in each samples.

A

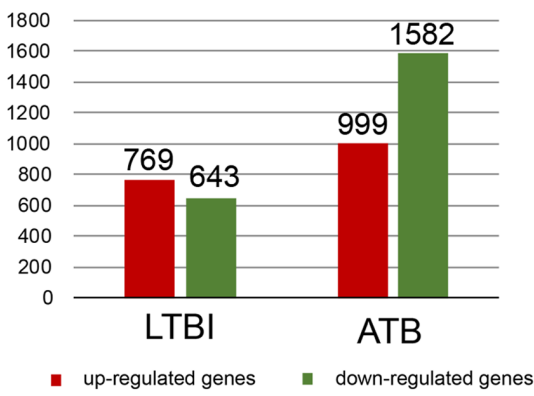

B

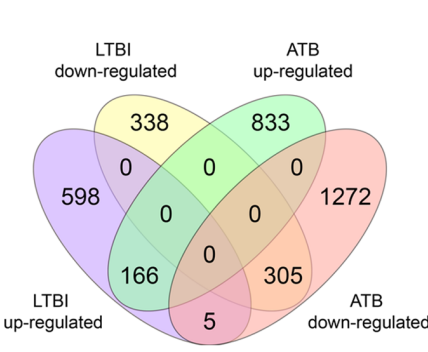

C

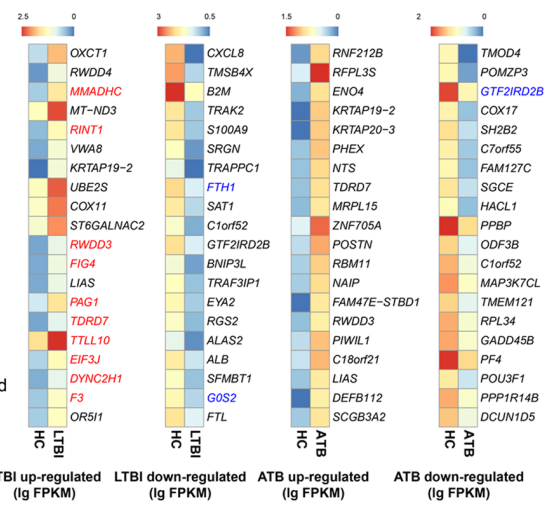

FIGURE 3 | Expression panels of serum exosomes for the LTBI and ATB samples. (A) The bar plot showed the numbers of DEGs in the LTBI and ATB compared with $\mathrm{HC}$. Red bars indicated the number of up-regulated genes in each sample, while the green bars indicated the number of down-regulated genes in each sample. (B) Venn diagram of DEGs showing the relatively distinct expression panels between the ATB and LTBI samples. (C) Heatmaps of top-20 DEGs in LTBI and ATB: colors indicate the estimate of base $10 \mathrm{log}$ ratio of expression levels that ranges from blue (low expression) to red (high expression). The three genes (labeled with blue color) showed the same down-regulated expression trends in both peripheral blood mononuclear cell (PBMC) and serum exosomes. The 10 up-regulated genes in LTBI (labeled with red color) were enriched in GO functions of protein binding or protein processing.

To illustrate the expression panels, the top-20 DEGs $(p<0.05)$ were selected (Figure $3 \mathrm{C}$ ), which might provide a potential panel for the differentiation of HC, LTBI, and ATB samples. Due to inadequate studies on serum exosomes of LTBI and ATB individuals, we compared our results with other studies performed in peripheral blood mononuclear cells (PBMCs) (Lee et al., 2016). Three genes (FTH1 and GOS2 in LTBI; GTF2IRD2B in ATB) shared the same down-regulated expression trends in PBMCs (Lee et al., 2016). However, there were some unmatched results, which increased in serum exosomes of LTBI but decreased in the serum cells of LTBI, e.g., RINT1 (Lee et al., 2016). This suggested that the contents of exosomes showed selective enrichment and secretion (Hannafon and Ding, 2013). Incidentally, 10 of the 20 up-regulated genes in LTBI were enriched in GO functions of protein binding or protein processing.

\section{Expression Patterns of Serum Exosomes in HC, LTBI, and ATB Individuals}

We classified all DEGs into six expression patterns according to the gene expression trends among the three samples (Figure 4A, Table 2, and Supplementary Table 3). 


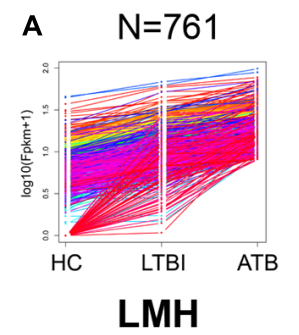

$N=1183$

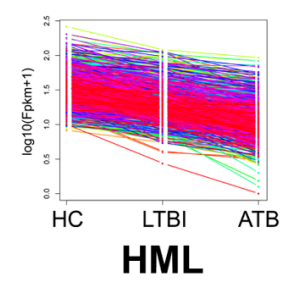

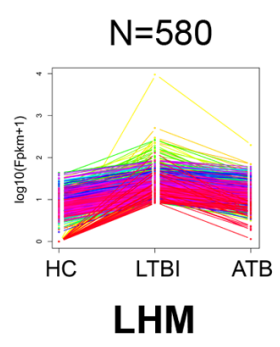

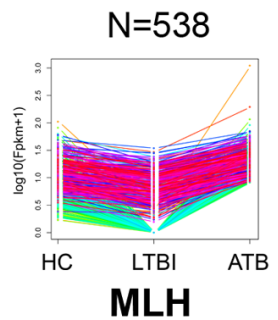

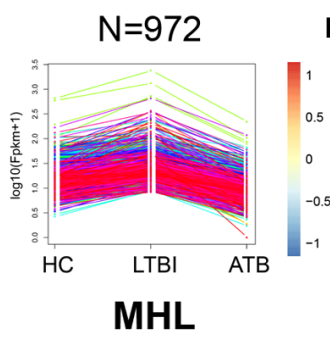

$\mathrm{N}=499$

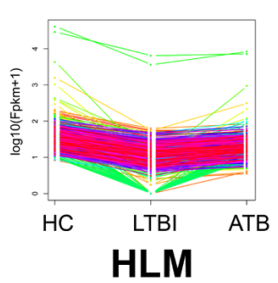

B

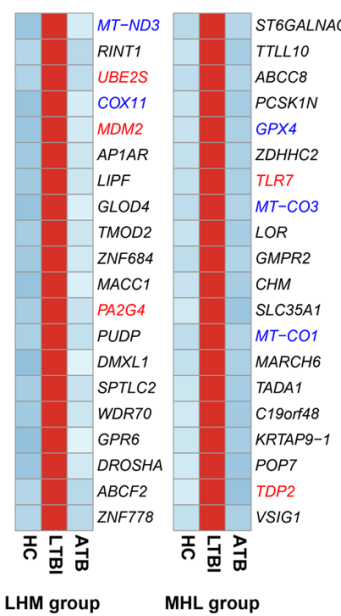

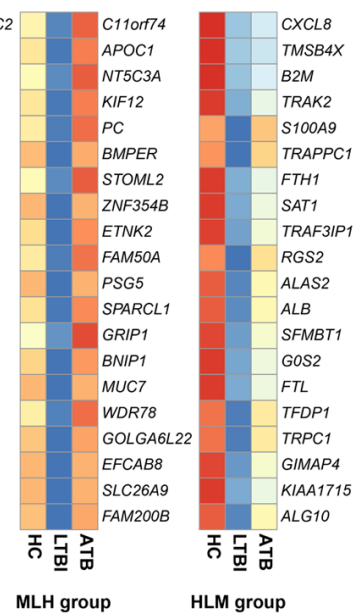

FIGURE 4 | The expression patterns of DEGs among the HC, LTBI, and ATB samples. (A) Six patterns were determined based on FPKM values, including LMH, LHM, MHL, HML, MLH, and HLM. (L, low expression level; M, medium expression level; H, high expression level; N, the gene numbers of each pattern).

(B) Heatmaps of top-20 high expressed and low expressed DEGs (LHM, MHL, MLH, and HLM patterns in the LTBI samples): colors indicate the estimate of base 10 log ratio of expression levels that ranges from blue (low expression) to red (high expression). The five genes (PA2G4, MDM2, UBE2S, TLR7, and TDP2, labeled with red color) in LHM and MHL groups were enriched in "cell cycle" functional category. The five genes (COX11, MT-ND3, MT-CO1, MT-CO3, and GPX4, labeled with blue color) were enriched in "mitochondria disorder" functional category.

TABLE 2 | The number of genes for six expression patterns.

\begin{tabular}{lc}
\hline Group & Number of genes \\
\hline LMH-group & 761 \\
HML-group & 1,183 \\
LHM-group & 580 \\
MHL-group & 972 \\
MLH-group & 538 \\
HLM-group & 499 \\
\hline
\end{tabular}

* L, low expression level; $M$, medium expression level; $H$, high expression level.

We further screened the top-20 genes in each pattern (Figure 4B and Supplementary Figure 2), especially those that were highly expressed in LTBI patients, which may provide potential targets of clinical diagnosis for these individuals. Five genes (COX11, MT-ND3, MT-CO1, MT-CO3, and GPX4) (Lee et al., 2009; Behar et al., 2011) with roles in mitochondrial disorder were highly expressed in LTBI patients. Previous studies have demonstrated that the integrity of the mitochondrial membrane is closely related to apoptosis, and is an effective anti-mycobacterial host defense mechanism (Ma et al., 2016). Interestingly, five genes (PA2G4, MDM2, UBE2S, TLR7, and TDP2) in both the LHM and MHL groups were identified as being enriched in the "cell cycle" functional category. As for the ATB highly expressed groups (Supplementary Figure 2), 12 genes displayed an increased trend and were in agreement with the previous studies (Pacis et al., 2015) (Supplementary Table 4). Among which, ACSL4 and CLU were related with lipid metabolic process (Kuch et al., 2014; Park et al., 2014), and MMRN1 and POSTN were relevant to cell adhesion (Adam et al., 2005; Michaylira et al., 2010). These results suggested that the top-20 genes for each pattern might provide potential molecular targets for the prevention, diagnosis, and treatment of LTBI and ATB individuals.

\section{Mtb RNAs Were Detected in LTBI Patients}

To investigate whether $M t b$ genes were secreted in serum exosomes, the sequencing data was mapped to the $M t b$ reference genome (NC00962). We identified 2, 1101, and 3 $M t b$ genes in $\mathrm{HC}$, LTBI, and ATB samples, respectively. The results showed $2 \mathrm{Mtb}$ rRNA genes ( $r r s$ and $r r l$ ) and $3 \mathrm{Mtb}$ genes ( $r r s, r r l$, and $R v 2917$ ) in HC and ATB samples, which were consistent with other studies where a few $M t b$ peptides were observed in ATB and HC individuals (Gutkin et al., 2016).

Importantly, many $M t b$ genes (1101) were discovered in LTBI exosomes. We further performed Cluster of Orthologous Group (COG) analysis for these and found a significant enrichment in COG category I "lipid transport and metabolism" (Figure 5). As for the expression of $M t b$ genes, we listed the top-20 expressed genes (evaluated by reads number, Supplementary Table 5), most of which were crucial genes for immunogenicity and virulence of $M t b$ (these included pks family, PPE family, and transpoases). Previous studies also indicated numerous $M t b$ proteins in exosomes from serum samples of LTBI individuals using Multiple Reaction Monitoring-Mass Spectrometry (MRM-MS) (Sinsimer et al., 2008). Comparing the results of the aforementioned study with ours, we found that eight $M t b$ genes were detected both in forms of protein and RNA (Supplementary Table 6). These results suggested that some $M t b$ cells in LTBI patients were disrupted and 


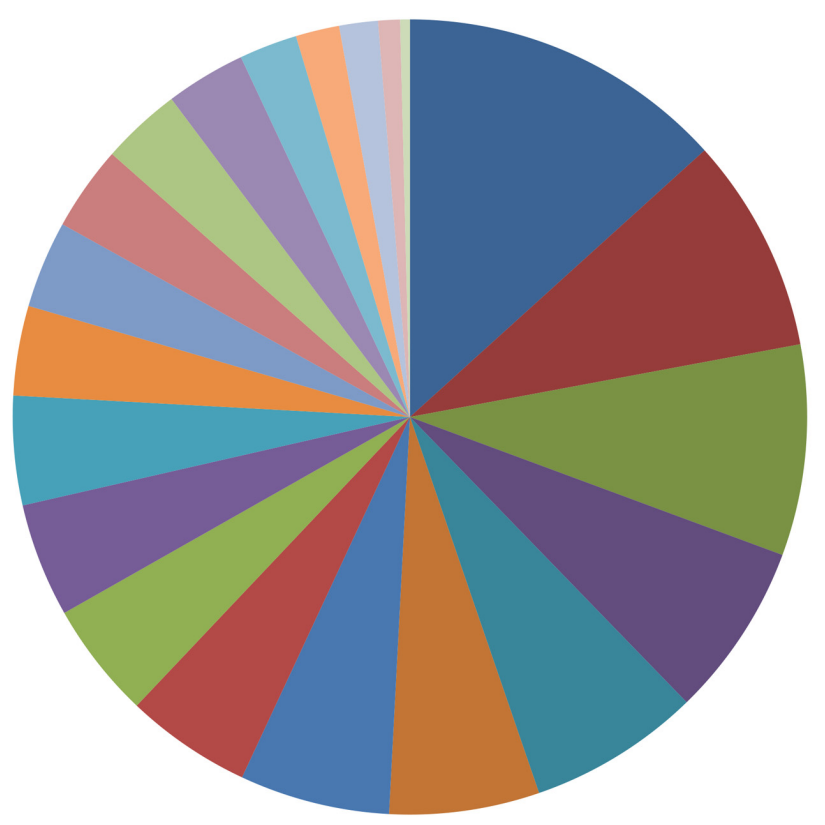

General function prediction only
Lipid transport and metabolism
Secondary metabolites biosynthesis, transport and catabolism
Energy production and conversion
Amino acid transport and metabolism
Replication, recombination and repair
Function unknown
Inorganic ion transport and metabolism
Cell wall/membrane/envelope biogenesis
Transcription
Carbohydrate transport and metabolism
Coenzyme transport and metabolism
Translation, ribosomal structure and biogenesis
Signal transduction mechanisms
Cell motility
- Posttranslational modification, protein turnover, chaperones
Nucleotide transport and metabolism
Defense mechanisms
Cell cycle control, cell division, chromosome partitioning
Intracellular trafficking, secretion, and vesicular transport
RNA processing and modification

FIGURE 5 | Mtb RNAs detected in HC, LTBI, and ATB. We identified 2, 1 101, and 3 Mtb genes in HC, LTBI, and ATB samples, respectively. The pie plot on the right panel indicated Cluster of Orthologous Group (COG) analysis on the 1101 Mtb genes in LTBI. A enrichment on COG category I "lipid transport and metabolism" was observed.

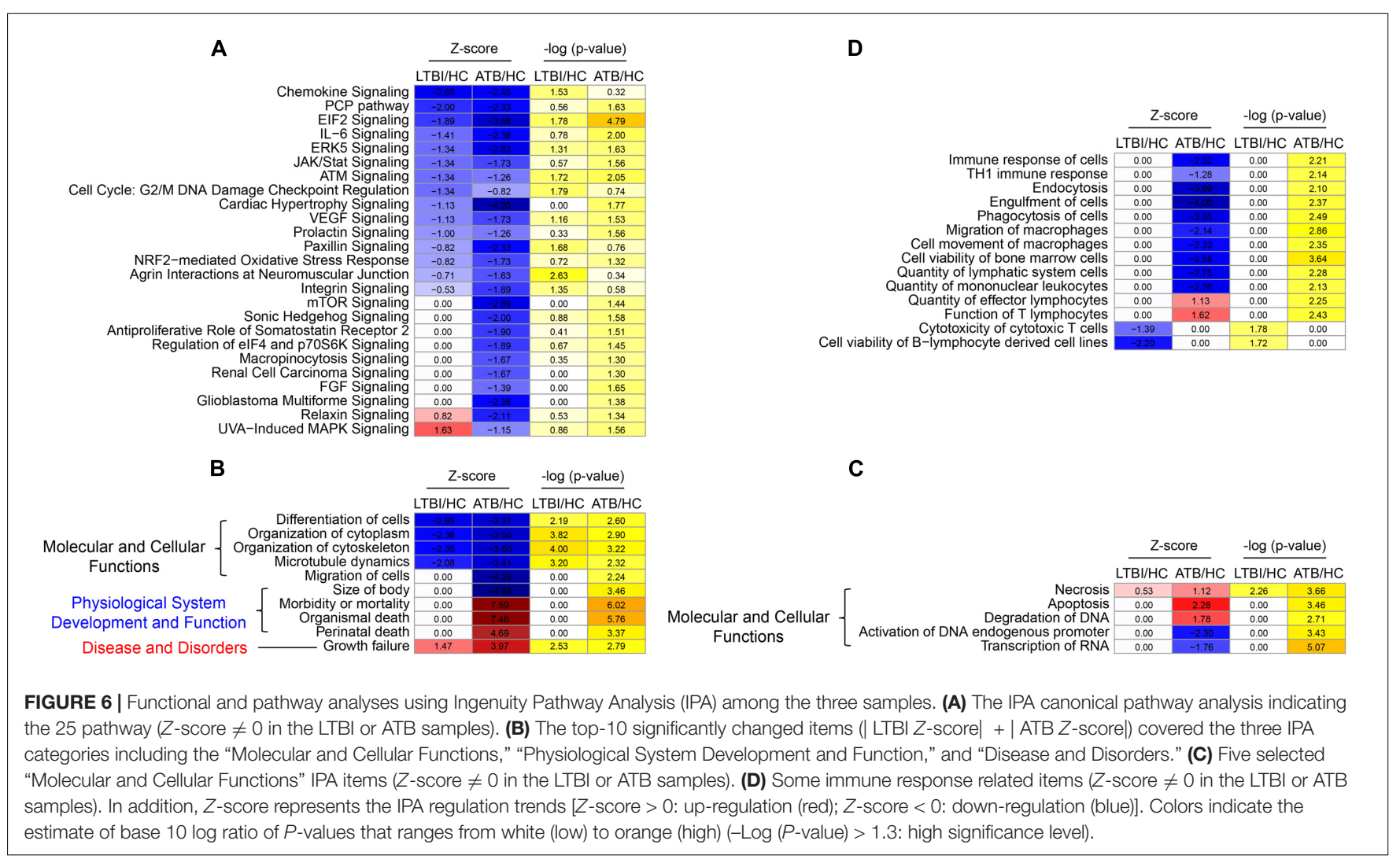


that many $M t b$ RNAs and proteins were exocytosed into the serum exosomes, although it was known that the $M t b$ cells in LTBI individuals were in a dormant state (Gengenbacher and Kaufmann, 2012).

\section{DISCUSSION}

In this research, we revealed distinct gene expression panels and patterns of serum exosomes from three groups, which included healthy, latently infected and actively infected TB individuals. (1) We identified the DEGs and screened the top-20 DEGs from the three groups; (2) we classified all the DEGs into six expression patterns and screened the top-20 genes from each pattern; (3) we identified numerous $M t b$ RNAs in the exosomes of LTBI patients. Our findings not only indicate the selective packaging of RNA cargoes into exosomes under different physiological status but also facilitate the development of potential targets for the diagnosis, prevention, and treatment of tuberculosis.

To further explore the mechanism of RNA packaging into exosomes under different infectious status, we performed functional and pathway analyses using IPA for the three samples (Supplementary Table 7). The IPA canonical pathway analysis indicated that the 25 signaling pathways were greatly suppressed in ATB samples (the second column of Figure 6A). Among these, the $P$-values of 20 items were less than 0.05 (the fourth column of Figure 6A). In the LTBI samples, relatively few pathways (15: the first column of Figure 6A) were suppressed, and 12 items demonstrated a weaker inhibitory effect than what was observed in the ATB samples. The inhibitory effect on signaling pathways in the exosomes increased with latent $\mathrm{TB}$ developing to the active disease, suggesting that different inhibition of cell activities occurs at different stages of $M t b$ infection.

Among all the IPA "Diseases and Bio Functions" items, the top-10 significantly changed items covered the three IPA categories including the "Molecular and Cellular Functions," "Physiological System Development and Function," and "Disease and Disorders" (Figure 6B). All categories displayed deteriorated trend in the ATB samples (the second column of Figure 6B), suggesting a systematic deteriorating state of the ATB patients. However, only five IPA functions (the first column of Figure 6A) exhibited a weaker trend in terms of deterioration in the LTBI samples, since most LTBI patients showed no symptoms of disease ${ }^{2}$. Additionally, five other IPA items from the "Molecular and Cellular Functions" category including "necrosis," "apoptosis," and "degradation of DNA" exhibited an overall deteriorated profile in the exosomes of ATB samples (the second column of Figure 6C), while it was only "necrosis" function that increased slightly in the LTBI samples (the first column of Figure 6C).

Cellular and humoral immunity plays a fundamental role in host defense against $M t b$ infection and dissemination (Zuñiga et al., 2012). According to the IPA "Diseases and Bio Functions" analysis, many immune response related items were significantly

${ }^{2}$ https://www.cdc.gov/tb/publications/ltbi/diagnosis.htm altered in serum exosome mRNA data (Figure 6D). Firstly, four types of items were suppressed in the ATB samples (the second column of Figure 6D), including: (1) immune response related functional items: "immune response of cells" and "Th1 immune response" functions, (2) phagocytosis related functional items: "endocytosis," "engulfment of cells," and "phagocytosis of cells," (3) cell movement related functional items: "migration of macrophages" and "cell movement of macrophages," and (4) other immune cell phenotype related functional items: "quantity of mononuclear leukocytes," "quantity of lymphatic system cells," and "cell viability of bone marrow cells functions." However, all the items in the LTBI samples were consistent with those in the HC samples (the first column of Figure 6D). In addition, the "quantity of effector lymphocytes" and "function of $\mathrm{T}$ lymphocytes" functions were up-regulated in the ATB samples, and remained unchanged in the LTBI samples. Finally, both the "cytotoxicity of cytotoxic $\mathrm{T}$ cells" and "cell viability of B-lymphocyte derived cell lines" functions significantly declined in the LTBI samples but remained constant in the ATB samples (Figure 6D).

Overall, our IPA analysis not only provided some potential biomarkers for the diagnosis of latent and active tuberculosis but also reflects some features suggestive of a gradual decline in health status as $M t b$ infection progresses.

\section{AUTHOR CONTRIBUTIONS}

LL and XZ conceived and designed the experiments. LL, XZ, JW, LP, HJ, and ZL performed the experiments. CL and ND analyzed the data. ZZ, FC, JZ, TC, and XJ provided suggestions on analysis. LL, CL, ND, and XZ wrote the paper, and FC revised the manuscript. All authors read and approved the final manuscript.

\section{ACKNOWLEDGMENTS}

This work was supported by National Natural Science Foundation of China (31500663, 31570133, 31600107, and 31601047); the "100-Talent Program" of Chinese Academy of Sciences (Y3CAS81554); Collaborative Innovation Center of Infectious diseases (PXM2015_014226_000058); the National Science and Technology Major Project of China (No. 2015ZX10004801-003 and 2016ZX10003001-011); the Beijing Natural Science Foundation (No. 5174035); the Collaborative Innovation Center of Infectious Diseases (No. PXM2015_014226_000058 and PXM2016_014226_000052); the Beijing Municipal Administration of Hospitals Clinical Medicine Development of Special Funding Support (No. ZYLX201304) and the Beijing Outstanding Talent Training Project (No. 2015000021469G188).

\section{SUPPLEMENTARY MATERIAL}

The Supplementary Material for this article can be found online at: http://journal.frontiersin.org/article/10.3389/fmicb.2017. 01051/full\#supplementary-material 


\section{REFERENCES}

Adam, F., Zheng, S., Joshi, N., Kelton, D. S., Sandhu, A., Suehiro, Y., et al. (2005). Analyses of cellular multimerin 1 receptors: in vitro evidence of binding mediated by alphaiibbeta3 and alphavbeta3. Thromb. Haemost. 94, 1004-1011. doi: 10.1160/TH05-02-0140

Alipoor, S. D., Mortaz, E., Garssen, J., Movassaghi, M., Mirsaeidi, M., and Adcock, I. M. (2016). Exosomes and exosomal mirna in respiratory diseases. Mediators Inflamm. 2016:5628404. doi: 10.1155/2016/5628404

Behar, S., Martin, C., Booty, M., Nishimura, T., Zhao, X., Gan, H., et al. (2011). Apoptosis is an innate defense function of macrophages against Mycobacterium tuberculosis. Mucosal Immunol. 4, 279-287. doi: 10.1038/mi. 2011.3

Bhatnagar, S., and Schorey, J. S. (2007). Exosomes released from infected macrophages contain Mycobacterium avium glycopeptidolipids and are proinflammatory. J. Biol. Chem. 282, 25779-25789. doi: 10.1074/jbc. M702277200

Bhatnagar, S., Shinagawa, K., Castellino, F. J., and Schorey, J. S. (2007). Exosomes released from macrophages infected with intracellular pathogens stimulate a proinflammatory response in vitro and in vivo. Blood 110, 3234-3244. doi: 10.1182/blood-2007-03-079152

Gengenbacher, M., and Kaufmann, S. H. E. (2012). Mycobacterium tuberculosis: success through dormancy. FEMS Microbiol. Rev. 36, 514-532. doi: 10.1111/j. 1574-6976.2012.00331.x

Gideon, H. P., and Flynn, J. L. (2011). Latent tuberculosis: what the host "sees"? Immunol. Res. 50, 202-212. doi: 10.1007/s12026-011-8229-7

Giri, P. K., Kruh, N. A., Dobos, K. M., and Schorey, J. S. (2010). Proteomic analysis identifies highly antigenic proteins in exosomes from $M$. tuberculosis-infected and culture filtrate protein-treated macrophages. Proteomics 10, 3190-3202. doi: 10.1002/pmic.200900840

Gusachenko, O. N., Zenkova, M. A., and Vlassov, V. V. (2013). Nucleic acids in exosomes: disease markers and intercellular communication molecules. Biochemistry 78, 1-7. doi: 10.1134/S000629791301001X

Gutkin, A., Uziel, O., Beery, E., Nordenberg, J., Pinchasi, M., Goldvaser, H., et al. (2016). Tumor cells derived exosomes contain hTERT mRNA and transform nonmalignant fibroblasts into telomerase positive cells. Oncotarget 7, 59173-59188. doi: 10.18632/oncotarget.10384

Hannafon, B. N., and Ding, W.-Q. (2013). Intercellular communication by exosome-derived micrornas in cancer. Int. J. Mol. Sci. 14, 14240-14269. doi: 10.3390/ijms140714240

Huang, D. W., Sherman, B. T., and Lempicki, R. A. (2009a). Bioinformatics enrichment tools: paths toward the comprehensive functional analysis of large gene lists. Nucleic Acids Res. 37, 1-13. doi: 10.1093/nar/ gkn923

Huang, D. W., Sherman, B. T., and Lempicki, R. A. (2009b). Systematic and integrative analysis of large gene lists using david bioinformatics resources. Nat. Protoc. 4, 44-57. doi: 10.1038/nprot.2008.211

Kaarteenaho-Wiik, R., Sademies, O., Pääkkö, P., Risteli, J., and Soini, Y. (2007) Extracellular matrix proteins and myofibroblasts in granulomas of sarcoidosis, atypical mycobacteriosis, and tuberculosis of the lung. Hum. Pathol. 38, 147-153. doi: 10.1016/j.humpath.2006.07.001

Kogure, T., Yan, I. K., Lin, W.-L., and Patel, T. (2013). Extracellular vesiclemediated transfer of a novel long noncoding rna tuc339: a mechanism of intercellular signaling in human hepatocellular cancer. Genes Cancer 4, 261-272. doi: 10.1177/1947601913499020

Kruh-Garcia, N. A., Wolfe, L. M., Chaisson, L. H., Worodria, W. O., Nahid, P., Schorey, J. S., et al. (2014). Detection of Mycobacterium tuberculosis peptides in the exosomes of patients with active and latent M. tuberculosis infection using MRM-MS. PLOS ONE 9:e103811. doi: 10.1371/journal.pone. 0103811

Kuch, E. M., Vellaramkalayil, R., Zhang, I., Lehnen, D., Brugger, B., Sreemmel, W., et al. (2014). Differentially localized acyl-CoA synthetase 4 isoenzymes mediate the metabolic channeling of fatty acids towards phosphatidylinositol. Biochim. Biophys. Acta 1841, 227-239. doi: 10.1016/j.bbalip.2013.10.018

Langmead, B., Trapnell, C., Pop, M., and Salzberg, S. L. (2009). Ultrafast and memory-efficient alignment of short DNA sequences to the human genome. Genome Biol. 10:R25. doi: 10.1186/gb-2009-10-3-r25
Lee, J., Hartman, M., and Kornfeld, H. (2009). Macrophage apoptosis in tuberculosis. Yonsei Med. J. 50, 1-11. doi: 10.3349/ymj.2009. 50.1 .1

Lee, S.-W., Wu, L. S.-H., Huang, G.-M., Huang, K.-Y., Lee, T.-Y., and Weng, J. T.-Y. (2016). Gene expression profiling identifies candidate biomarkers for active and latent tuberculosis. BMC Bioinformatics 17(Suppl. 1):3. doi: 10.1186/s12859015-0848-X

Ma, J., Tian, M., Fan, X., Yu, Q., Jing, Y., Wang, W., et al. (2016). Mycobacterium tuberculosis multistage antigens confer comprehensive protection against preand post-exposure infections by driving th1-type t cell immunity. Oncotarget 7, 63804-63815. doi: 10.18632/oncotarget.11542

Michaylira, C. Z., Wong, G. S., Miller, C. G., Gutierrez, C. M., Nakagawa, H., Hammond, R., et al. (2010). Periostin, a cell adhesion molecule, facilitates invasion in the tumor microenvironment and annotates a novel tumor-invasive signature in esophageal cancer. Cancer Res. 70, 5281-5292. doi: 10.1158/00085472.CAN-10-0704

Ouellet, H., Johnston, J. B., and de Montellano, P. R. (2011). Cholesterol catabolism as a therapeutic target in Mycobacterium tuberculosis. Trends Microbiol. 19, 530-539. doi: 10.1016/j.tim.2011.07.009

Pacis, A., Tailleux, L., Lambourne, A. M. M., Maclsaac, J. L., Yotova, V., Dumaine, A., et al. (2015). Bacterial infection remodels the DNA methylation landscape of human dendritic cells. Genome Res. 25, 1801-1811. doi: 10.1101/ gr.192005.115

Park, S., Mathis, K. W., and Lee, I. K. (2014). The physiological roles of apolipoprotein J/clusterin in metabolic and cardiovascular diseases. Rev. Endocr. Metab. Disord. 15, 45-53. doi: 10.1007/s11154-0139275-3

Shammari, B. A., Shiomi, T., Tezera, L., Bielecka, M. K., Workman, V., Sathyamoorthy, T., et al. (2015). The extracellular matrix regulates granuloma necrosis in tuberculosis. J. Infect. Dis. 212, 463-473. doi: 10.1093/infdis/ jiv076

Singh, P. P., LeMaire, C., Tan, J. C., Zeng, E., and Schorey, J. S. (2011). Exosomes released from $M$. tuberculosis infected cells can suppress IFN-gamma mediated activation of naive macrophages. PLOS ONE 6:e18564. doi: 10.1371/journal. pone.0018564

Singh, P. P., Li, L., and Schorey, J. S. (2015). Exosomal RNA from Mycobacterium tuberculosis-infected cells is functional in recipient macrophages. Traffic 16, 555-571. doi: 10.1111/tra.12278

Sinsimer, D., Huet, G., Manca, C., Tsenova, L., Koo, M.-S., Kurepina, N., et al. (2008). The phenolic glycolipid of Mycobacterium tuberculosis differentially modulates the early host cytokine response but does not in itself confer hypervirulence. Infect. Immun. 76, 3027-3036. doi: 10.1128/IAI. 01663-07

Subramanian, A., Gupta, V., Sarkar, S., Maity, G., Banerjee, S., Ghosh, A., et al. (2016). Exosomes in carcinogenesis: molecular palkis carry signals for the regulation of cancer progression and metastasis. J. Cell Commun. Signal. 10, 241-249. doi: 10.1007/s12079-016-0338-6

Théry, C. (2011). Exosomes: secreted vesicles and intercellular communications. F1000 Biol. Rep. 3:15. doi: 10.3410/B3-15

Trapnell, C., Pachter, L., and Salzberg, S. L. (2009). Tophat: discovering splice junctions with rna-seq. Bioinformatics 25, 1105-1111. doi: 10.1093/ bioinformatics/btp120

Trapnell, C., Williams, B. A., Pertea, G., Mortazavi, A., Kwan, G., Baren, M. J. V., et al. (2010). Transcript assembly and quantification by rna-seq reveals unannotated transcripts and isoform switching during cell differentiation. Nat. Biotechnol. 28, 511-515. doi: 10.1038/nbt.1621

Tufarielloa, J. M., Chana, J., and Flynn, J. L. (2003). Latent tuberculosis: mechanisms of host and bacillus that contribute to persistent infection. Lancet Infect. Dis. 3, 578-590. doi: 10.1016/s1473-3099(03)00741-2

Valadi, H., Ekström, K., Bossios, A., Sjöstrand, M., Lee, J. J., and Lötvall, J. O. (2007). Exosome-mediated transfer of mRNAS and microRNAs is a novel mechanism of genetic exchange between cells. Nat. Cell Biol. 9, 654-659. doi: 10.1038/ ncb1596

Velayati, A. A., Abeel, T., Shea, T., Konstantinovich Zhavnerko, G., Birren, B., Cassell, G. H., et al. (2016). Populations of latent Mycobacterium tuberculosis lack a cell wall: isolation, visualization, and whole-genome characterization. Int. J. Mycobacteriol. 5, 66-73. doi: 10.1016/j.ijmyco.2015.12.001 
Zuñiga, J., Torres-García, D., Santos-Mendoza, T., Rodriguez-Reyna, T. S., Granados, J., and Yunis, E. J. (2012). Cellular and humoral mechanisms involved in the control of tuberculosis. Clin. Dev. Immunol. 2012:193923. doi: $10.1155 / 2012 / 193923$

Conflict of Interest Statement: The authors declare that the research was conducted in the absence of any commercial or financial relationships that could be construed as a potential conflict of interest.
Copyright (c) $2017 \mathrm{Lv}$, Li, Zhang, Ding, Cao, Jia, Wang, Pan, Jia, Li, Zhang, Chen and Zhang. This is an open-access article distributed under the terms of the Creative Commons Attribution License (CC BY). The use, distribution or reproduction in other forums is permitted, provided the original author(s) or licensor are credited and that the original publication in this journal is cited, in accordance with accepted academic practice. No use, distribution or reproduction is permitted which does not comply with these terms. 